\title{
An improvement of GPS height estimations: stochastic modeling
}

\author{
Shuanggen Jin ${ }^{1,2,3}$, J. Wang ${ }^{2}$, and Pil-Ho Park ${ }^{1}$ \\ ${ }^{1}$ Space Geodesy Research Group, Korea Astronomy and Space Science Institute, Daejeon 305-348, South Korea \\ ${ }^{2}$ School of Surveying and Spatial Information Systems, University of New South Wales, Sydney, NSW 2052, Australia \\ ${ }^{3}$ Shanghai Astronomical Observatory, Chinese Academy of Sciences, Shanghai 200030, China
}

(Received November 12, 2004; Revised March 11, 2005; Accepted March 14, 2005)

\begin{abstract}
The results of GPS positioning depend on both functional and stochastic models. In most of the current GPS processing programs, however, the stochastic model that describes the statistical properties of GPS observations is usually assumed that all GPS measurements have the same accuracy and are statistically independent. Such assumptions are unrealistic. Although there were only a few studies modeling the effects on the GPS relative positioning, they are restricted to short baselines and short session lengths. In this paper, the stochastic modeling for IGS long-baseline positioning (with 24-hour session) is analyzed in the GAMIT software by modified stochastic models. Results show that any mis-specifications of stochastic model result in unreliable GPS baseline results, and the deviation of baseline estimations reaches as much as $2 \mathrm{~cm}$ in the height component. Using the stochastic model of satellite elevation angle-based cosine function, the precision of GPS baseline estimations can be improved, and the GPS baseline component is closest to the reference values, especially GPS height.
\end{abstract}

Key words: Stochastic modeling, GPS, IGS, height.

\section{Introduction}

The global positioning system (GPS) has become an essential tool for the high-precise positioning. GPS height, however, is still poorly estimated. Traditionally, data processing for precise GPS positioning is invariably performed using the least squares (LS) method. The quality of LS solutions depends on the model's formulation regarding GPS observations, including both a functional model, which describes the mathematical relationships between the GPS measurements and unknown parameters, and a stochastic model, which tells us the statistical properties of the measurements (Han and Rizos, 1995; Rizos, 1997; Brunner et al., 1999). Over the past two decades, the functional models for GPS measurements have been investigated in considerable detail. As GPS measurement errors are dominated by the systematic errors caused by the multi-path, atmosphere, orbit effects, and so on, it is impossible to model all systematic errors in the functional model, especially in the vertical direction. Therefore, modeling some systematic errors into the stochastic model is a current challenging topic to further realize the full potential of increasingly more accurate GPS positioning, especially GPS height.

To date, however, accurate stochastic modeling for the GPS measurements has not sufficiently been analyzed. In the current stochastic models for GPS positioning, it is usually expressed with a simple format in most of the GPS processing programs, assuming that all the GPS measurements have the same variance. The time-invariant covariance matrix of the double-differenced (DD) measurements

Copy right(c) The Society of Geomagnetism and Earth, Planetary and Space Sciences (SGEPSS); The Seismological Society of Japan; The Volcanological Society of Japan; The Geodetic Society of Japan; The Japanese Society for Planetary Sciences; TERRAPUB is then constructed using the error propagation law. Such assumptions are unrealistic (e.g. Wang, 1998a; Wang et al., 1998b; Bona, 2000). Any mis-specifications of stochastic model will result in unreliable parameter estimations. Many researchers are aware of the importance of the stochastic model. Blewitt (1998) pointed out that both stochastic and functional models are equivalent in the theory.

Although there were only a few studies modeling the effects on the GPS relative positioning (El-Rabbany, 1994; Wang et al., 1998b, 2002; Satirapod et al., 2002, 2003; Tiberius and Kenselaar, 2003), they were restricted to short baselines and short session lengths.

In this paper, the stochastic modeling for IGS longbaseline positioning (with 24-hour session) is analyzed in the GAMIT software modified with stochastic models, and the impact on GPS baseline estimations, especially GPS height, is investigated. In the following section the stochastic modeling methods and results for IGS station positioning are presented.

\section{Stochastic Modeling Methods and Results}

The linear observation equations of GPS can be expressed as

$$
L=A x+v
$$

where $L$ is a vector of the observed-minus-computed DD carrier phase values (O-C), $A$ is the design matrix, $x$ is the unknown parameters and $v$ is the residual. Using the least square method (LS), the unknown parameters and accuracy indicator can be obtained, namely

$$
\begin{aligned}
& \hat{x}=\left(A^{T}\left(C_{x}\right)^{-1} A\right)^{-1} A^{T}\left(C_{x}\right)^{-1} L \\
& v=L-A \hat{x} \\
& \hat{\sigma}^{2}=\frac{v^{T}\left(C_{x}\right)^{-1} v}{f}
\end{aligned}
$$


where $\hat{x}$ is the parameter estimate, $C_{x}$ is the variancecovariance matrix for the double-differenced GPS measurements, called the stochastic model, $\hat{\sigma}$ is the accuracy indicator and $f$ is the degree of freedom. It's easy to see that the estimation of unknown parameter $x$ and its accuracy indicator are dependent on the stochastic model. Any misspecifications of stochastic model will result in unreliable unknown-parameter estimations. In our initial studies, the models to be considered here are the ones that can be easily implemented within scientific GPS data processing software packages such as GAMIT and BERNESE. The details of the variations in the stochastic models are discussed below.

\subsection{Standard stochastic model}

In a commonly-used stochastic model, it is usually assumed that all the carrier phases or pseudo-ranges have the same variance $\left(\sigma^{2}\right)$ and are statistically independent. Therefore, the observation $\Phi$ is treated as independent and uncorrelated, and the covariance matrix of the observation $\Phi$ can be formulated as:

$$
\operatorname{Cov}(\Phi)=\sigma^{2} I
$$

where $I$ is the unit matrix. Through the error propagation law the time-invariant covariance matrix (called the stochastic model) of the DD measurements can be obtained.

$$
C_{x}=\sigma^{2}\left[\begin{array}{cccc}
4 & 2 & \cdots & 2 \\
2 & 4 & \cdots & 2 \\
\vdots & \vdots & \ddots & 2 \\
2 & 2 & \cdots & 4
\end{array}\right]
$$

This is a standard stochastic model for DD measurements, which is easy to implement in practice. However, this simplified stochastic model may contain some misspecifications, and thus could result in unreliable GPS positioning results.

\subsection{Baseline length dependent weighting}

As the distances between GPS stations in a network are different, the baseline length dependent variances for GPS measurements are defined with the following function (King and Bock, 1999):

$$
\sigma^{2}=\alpha^{2}+\beta^{2} * \text { Distance }^{2}
$$

where $\alpha=9 \mathrm{~mm}$ and $\beta=0.1 \mathrm{~mm} / \mathrm{km}$. This formula describes the relative qualities of GPS measurements from different GPS baselines in a network. And the baseline components may be treated as observations with defined uncertainty to improve the geometry of the network solutions for the parameter estimation.

\subsection{Satellite elevation angle dependent weighting}

GPS measurement errors are dominated by systematic errors, such as signal-to-noise ratio, atmospheric delay and multi-path errors, which may be closely connected with the satellite elevation angles and azimuths. The effects of these error sources are different for each satellite. Therefore GPS measurements from different satellites may not have the same accuracy. In order to model the variances of GPS measurements from different satellites, a function of satellite elevation angle is used to describe the variances of raw GPS measurements in practice, namely:

$$
\sigma_{\phi_{r}^{s}(i)}^{2}=a^{2}+b^{2} * f^{2}\left(\operatorname{elev}_{r}^{j}(i)\right)
$$

where $a$ and $b$ are constant values, and $f\left(\operatorname{elev}_{r}^{j}(i)\right)$ is the function of satellite elevation angle at epoch $i$. Given the variances of one-way GPS measurements, the covariance matrix for the DD measurements is derived using the error propagation law:

$$
C_{L(i)}=a^{2} \cdot T_{a i}+b^{2} \cdot T_{b i}
$$

where $T_{a i}$ is the same as Eq. (4), and

$$
T_{b i}=\left[\begin{array}{cccc}
f_{1 i}+f_{2 i} & f_{1 i} & \cdots & f_{1 i} \\
f_{1 i} & f_{1 i}+f_{3 i} & \cdots & f_{1 i} \\
\vdots & \vdots & \ddots & \vdots \\
f_{1 i} & f_{1 i} & \cdots & f_{1 i}+f_{n i}
\end{array}\right]
$$

where

$$
f_{j i}=f\left(\operatorname{elev}_{1}^{j}(i)\right)+f\left(\operatorname{elev}_{s}^{j}(i)\right), \quad j=1,2, \ldots, n .
$$

Because of the complexity of unknown factors in the stochastic modeling, the functional relationship between the accuracy of GPS measurements and satellite elevation angles can only be approximately expressed. The sine and cosine functions of satellite elevation angles are often used for this purpose. In the GAMIT software package the satellite elevation angle-based sine function is currently used to calculate the accuracy of the one-way GPS measurements (King and Bock, 1999):

$$
\sigma_{\phi_{r}^{s}(i)}^{2}=a^{2}+b^{2} / \sin ^{2}\left(\operatorname{elev}_{r}^{j}(i)\right)
$$

where $a=4.3 \mathrm{~mm}$ and $b=3 \mathrm{~mm}$. The function is a good approximation to the tropospheric sensitivity. In addition, the cosine function of the satellite elevation angle is usually used to calculate the accuracy of the one-way GPS measurements (Jin and Jong, 1996; Barnes et al., 1998; Hugentobler et al., 2001; Jin and Wang, 2004), expressed by the following two formulas

$$
\begin{aligned}
\sigma_{\phi_{r}^{s}(i)}^{2} & =a^{2}+b^{2} / \cos ^{2}\left(\operatorname{elev}_{r}^{j}(i)\right) \\
\sigma_{\phi_{r}^{s}(i)}^{2} & =a^{2}+b^{2} / \cos ^{-2}\left(\operatorname{elev}_{r}^{j}(i)\right)
\end{aligned}
$$

For this study, the GAMIT software package has been modified to include the two functions as one of the options for stochastic modeling, and other rarely used functions of the satellite elevation angle are not discussed here.

The test data sets used were from the Australian IGS GPS network (Fig. 1). The GAMIT software was used for the data processing with constrained positions of the IGS stations (horizontal and vertical coordinates were assigned a standard derivation of $5 \mathrm{~mm}$ and $10 \mathrm{~mm}$, respectively). The IGS precise orbits, phase center variation model of GPS antenna and ERP (Earth Rotation Parameters) were used. Some parameters used in the data processing were: 1) cut-off elevation: 15 degrees; and 2) GPS data sampling interval: 30 seconds.

The performance of the following five stochastic modeling methods for GPS measurements was evaluated: 


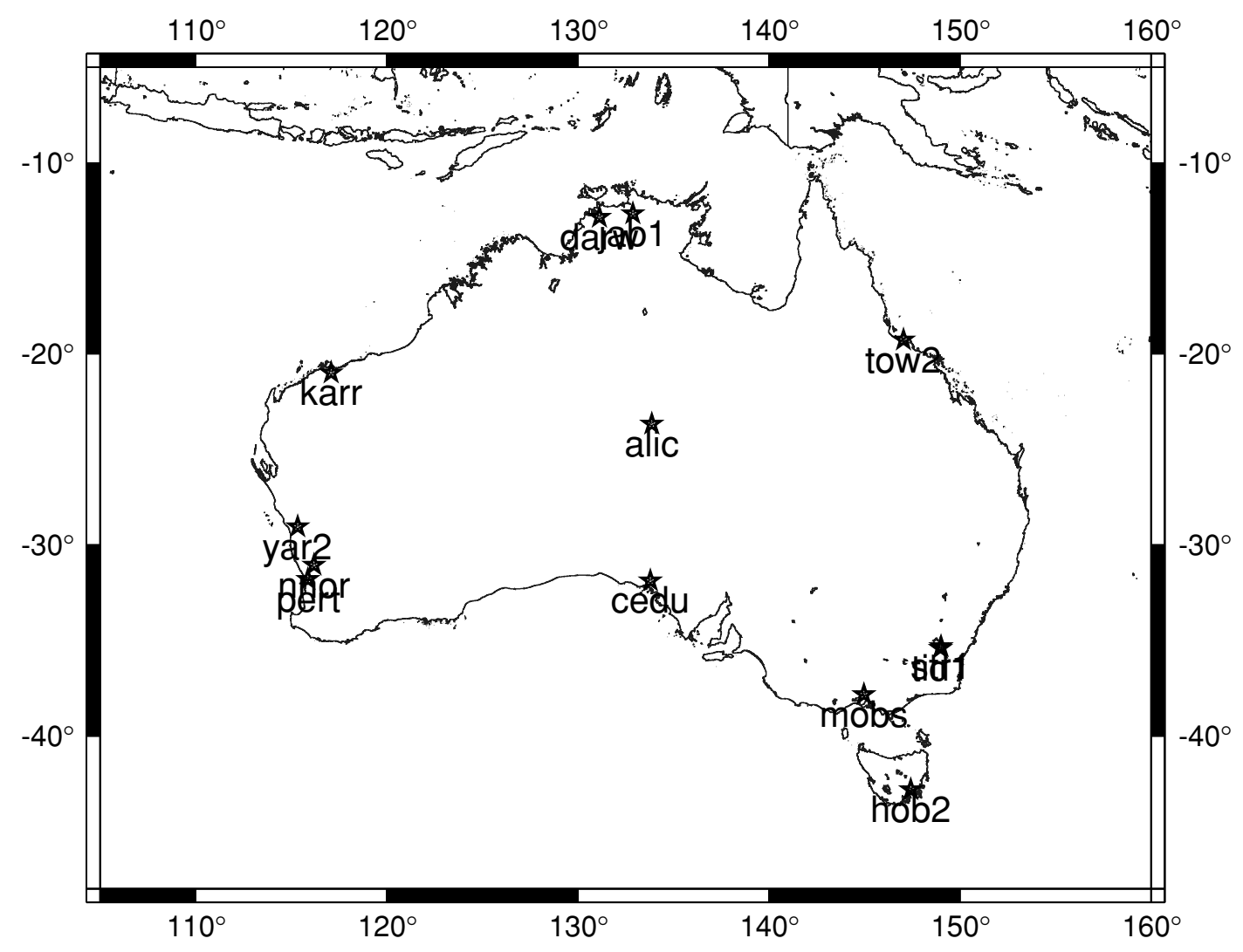

Fig. 1. The distribution of GPS stations in Australia.

A: Standard stochastic model;

B: Baseline length dependent weighting;

C: Satellite elevation angle-based sine function;

D: Satellite elevation angle-based cosine function, expressed as Eq. (10); and

E: Satellite elevation angle-based secant function expressed as Eq. (11).

Figure 2 shows the standard deviations of each baseline component using different stochastic models, and Fig. 3 shows the deviations between estimated baseline components and referenced values on the day 300, 2003. The reference value is the fitting solution of 14-year daily position estimates of IGS stations processed by GIPSY in the ITRF2000 (International Terrestrial Reference Frame 2000) (http://sideshow.jpl.nasa.gov/mbh/all/table.txt). Here, we didn't directly compare stochastic modeling solutions between GAMIT and GIPSY as both softwares have different functional and stochastic models. For instance, the GAMIT software uses GPS double difference (DD) based on the least squares (LS) method, and the GIPSY software uses GPS non-difference based on the Kalman Filtering technique. In addition, GIPSY doesn't have a set stochastic model. We just used the reference value, which is actually a good average value of long-term GPS solutions processed by GIPSY. The comparisons in Figs. 2 and 3 show that, using different stochastic models, the differences between the standard deviations reach $1-2 \mathrm{~mm}$ in horizon- tal components and 2 3 $\mathrm{mm}$ in height, while the differences between the baseline estimations are up to $20 \mathrm{~mm}$ in height components. In addition, the standard deviations of the GPS baseline estimations based on the Method B are higher than those from the standard processing method (A), and the satellite elevation angle-based cosine functions (Method D and E) are better than other tested stochastic models. Although the standard deviations of methods $\mathrm{C}$ and $\mathrm{E}$ are small, the corresponding baseline components severely deviate from the reference values. The traditionally used stochastic model in the GAMIT program, satellite elevation angle-based sine function (Method C) not only degrades the precision of GPS baseline estimations, but also result in a big deviation from the reference value. Comparing the reference value, the baseline components of method $\mathrm{D}$ is closest to the reference value, especially GPS height. Therefore, among all the tested stochastic modeling methods, Method D has the best performance.

Figures 4 and 5 are the results on 301, 2003. Among all the tested stochastic modeling methods, Method D has the best performance. The precision of GPS baseline estimations can be improved, and the GPS baseline component, especially GPS height is closest to the reference values. GPS data on another day $(151,2004)$ are further tested, and the conclusions are the same.

\section{Discussions}

The GPS baseline or coordinate was usually estimated under the assumption that all the GPS measurements have the same variance. Such assumptions are unrealistic. As 

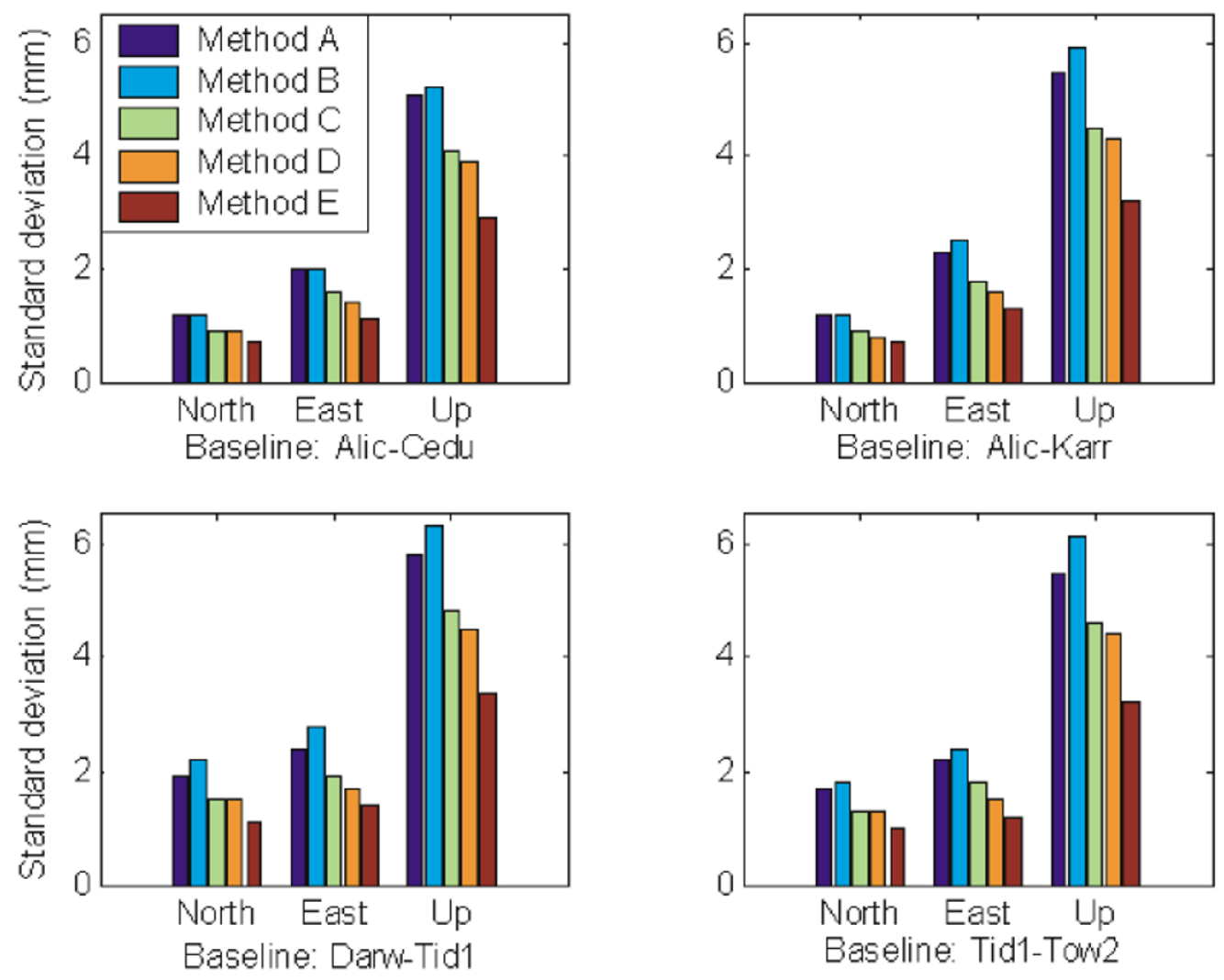

Fig. 2. Comparisons of baseline standard deviations on 300, 2003.
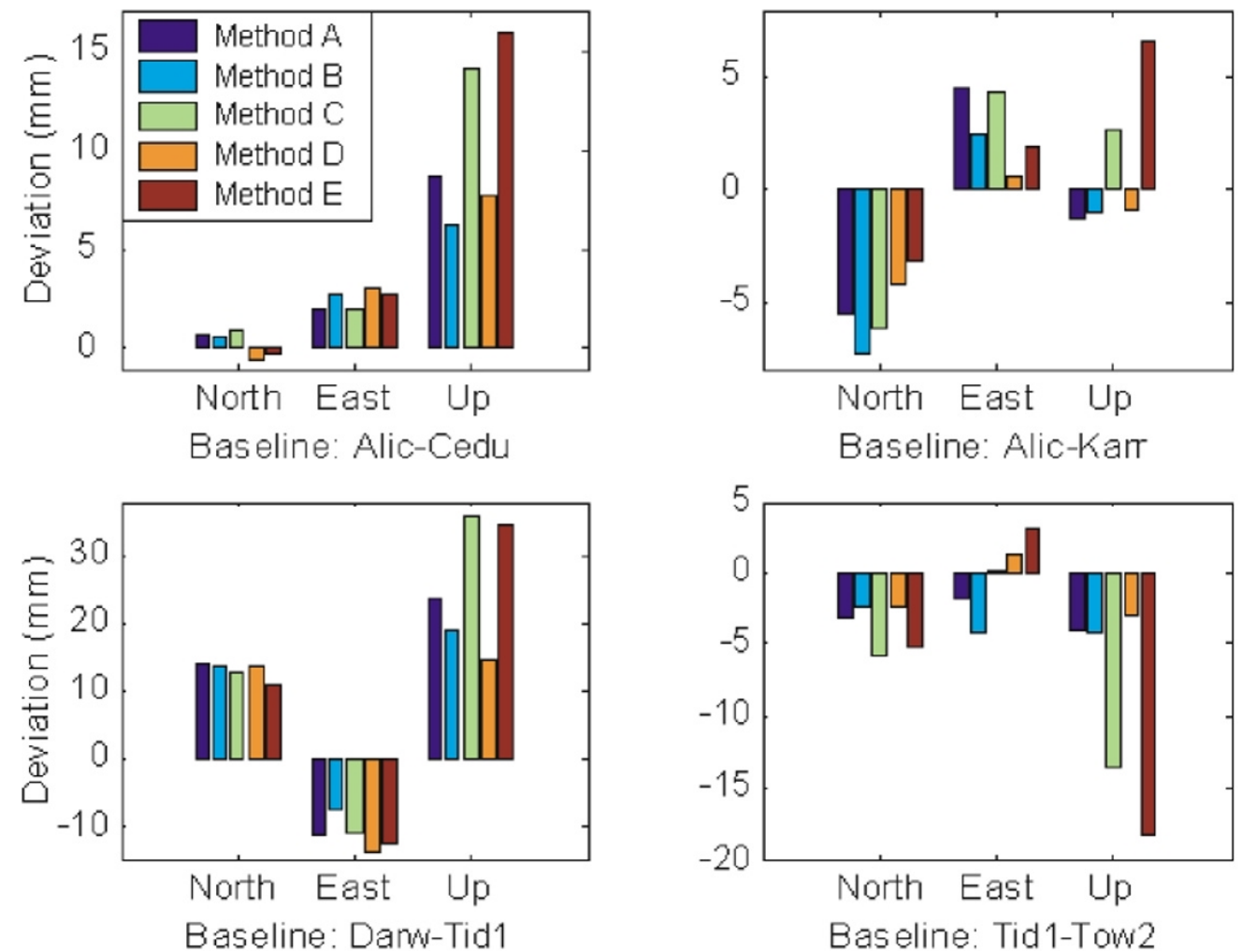

Fig. 3. Deviations between estimated baseline components and reference values on 300, 2003. 

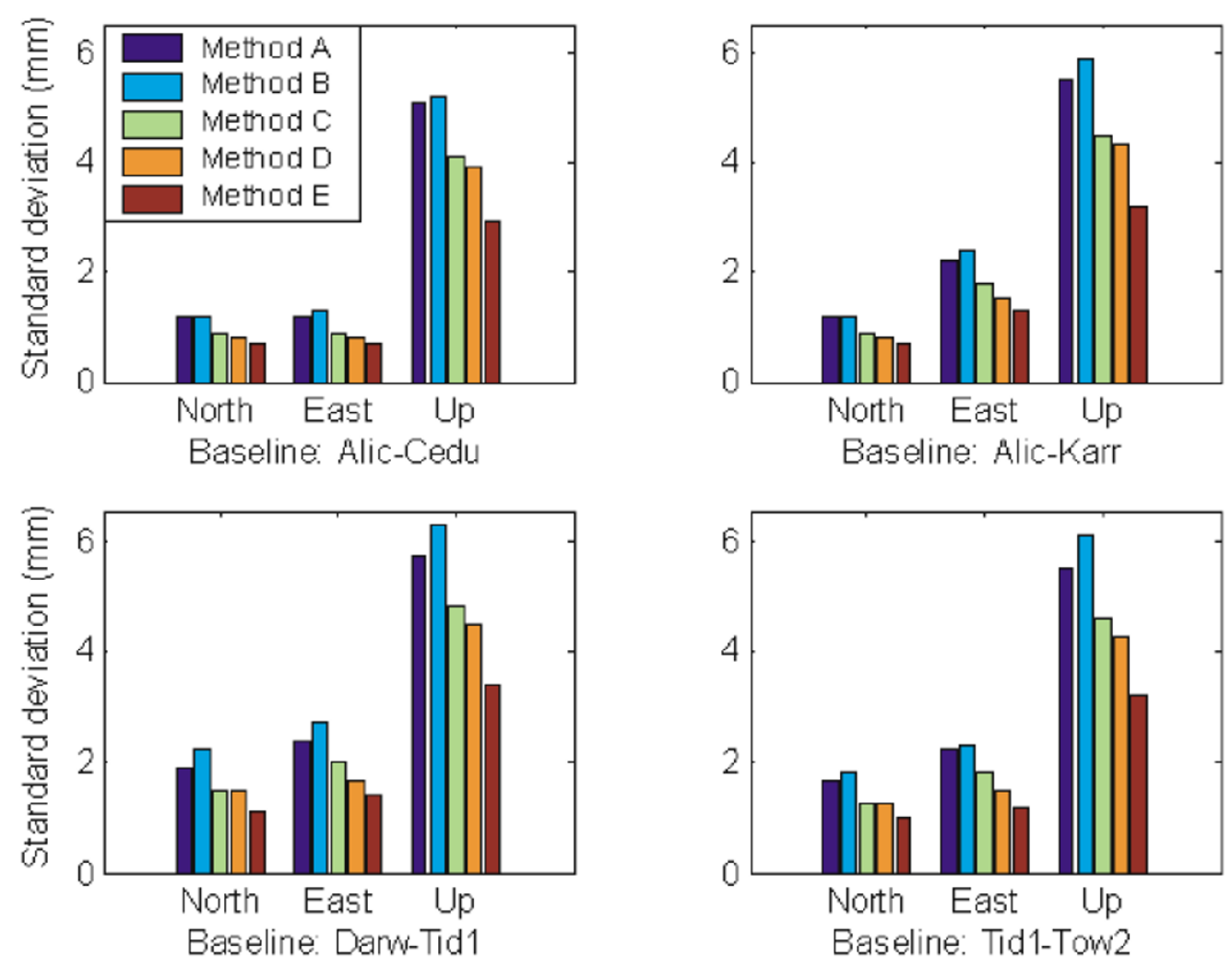

Fig. 4. Comparisons of baseline standard deviations on 301, 2003.
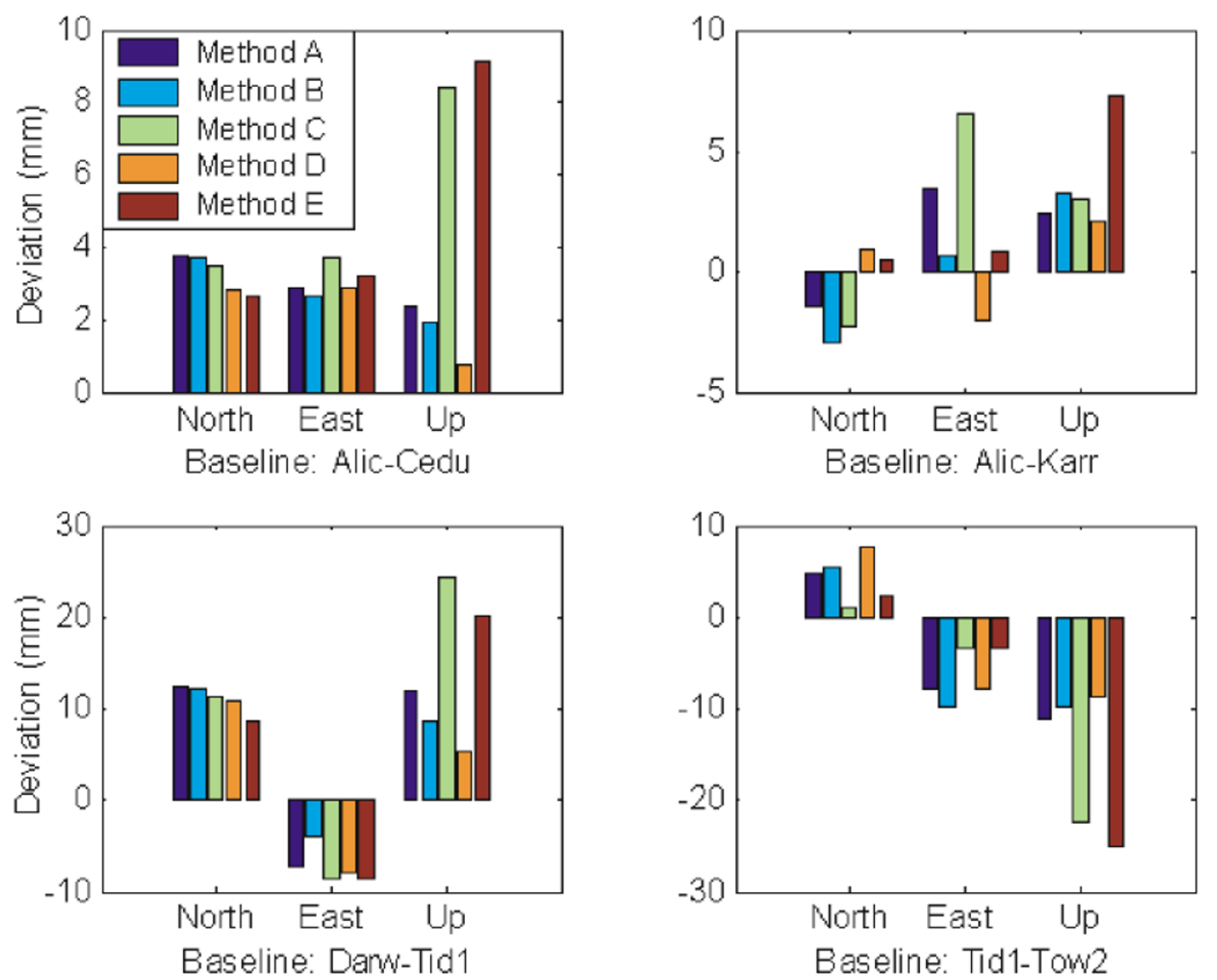

Fig. 5. Deviations between estimated baseline components and referenced values on 301, 2003. 
GPS measurement errors are dominated by the systematic errors caused by the multi-path, atmosphere, receiver noise and orbit effects, which are quite different for each satellite. Therefore the measurements obtained from different satellites cannot have the same accuracy due to varying noise levels. Although some errors can be mitigated or minimized by some models and appropriate processing techniques, the most important error sources in a low GPS satellite elevate angle are not well eliminated, such as the tropospheric correction models of Saastamoinent (1973) and Hopfield (1969). Figure 6 shows the relationship between DD residuals and the satellite elevation angles. The lower the satellite elevation angles, the higher the DD residuals, which were mainly caused by the multi-path and troposheric delay.

Both errors increase when the satellite elevation cut-off angle decreases. Such errors are not good but difficult to be taken into account in the functional models. Therefore, in the theory, it can further improve accurate GPS positioning (especially GPS height) by modeling some systematic errors into the stochastic model.

The test results show that using the stochastic model of satellite elevation angle-based cosine function, not only the precision is obviously improved, but also the GPS baseline component, especially GPS height, is closest to the reference value. The reference value is the fitting solution of 14-year daily position estimates of IGS stations processed by GIPSY (http://sideshow.jpl.nasa.gov/mbh/all/table.txt). We also take another reference value from ITRF2000 solution published by IERS, namely the combination solution of each GPS analysis center (e.g. GFZ, JPL, CODE, IGN, and NOAA) (http://itrf.ensg.ign.fr/ITRF_solutions/2000/ results/ITRF2000_GPS.SSC.txt). And the comparison conclusion is the same, which the stochastic model of satellite elevation angle-based cosine function has the best performance.

It shows that other tested stochastic models cannot well model some systematic errors, and the cosine weight function is sensitive to errors with elevation angle variations. In addition, the impact of stochastic modeling on GPS base-
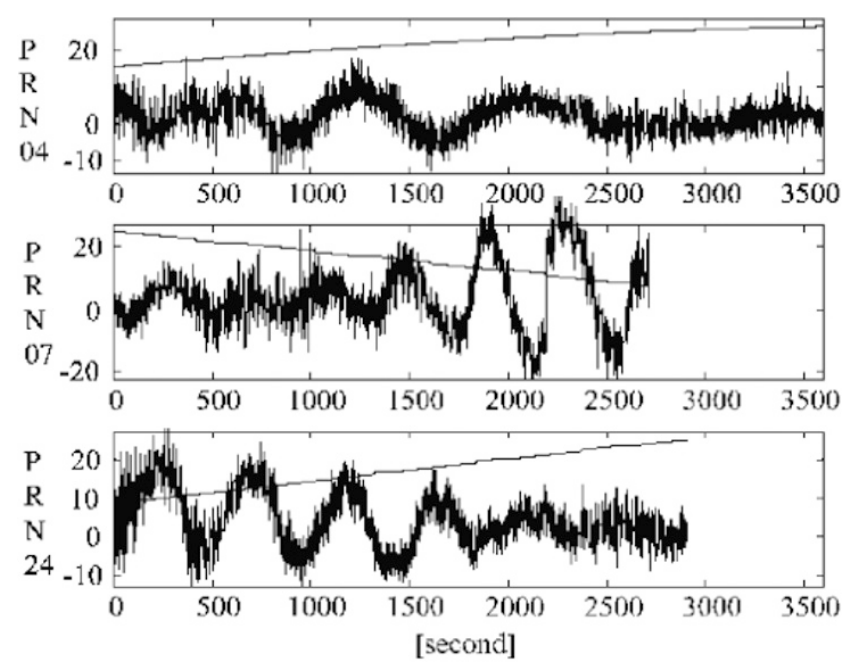

Fig. 6. Satellite elevation angle (line, in degrees) and DD residuals (curvy line, in $\mathrm{mm}$ ) with respect to time. line estimates with different cutoff elevation angles, $8^{\circ}, 10^{\circ}$, $15^{\circ}$, and $20^{\circ}$, is further tested and the cosine weighting can get better solutions when weighting more on low elevation data. This result is also supported by daily repeatabilities.It shows that the cosine weighting can further improve GPS positioning solutions by modeling some unmodeled errors.

This initial study has demonstrated that the stochastic model methods play an important role in the GPS height estimation. Suitable stochastic modeling strategies for GPS measurements and baseline components (or the coordinates of GPS tracking stations) should be further investigated.

\section{Concluding Remarks}

It has been noted that, in the paper, with different stochastic modeling methods tested in the GAMIT, the changes in the estimated baseline values can reach $2 \mathrm{~cm}$ in height components, and mis-specification in the stochastic models will result in unreliable baseline estimations. The traditional stochastic model used in the GAMIT program, satellite elevation angle-based sine function, not only degrades the precision of GPS baseline estimations, but also will result in a big deviation (especially height component) from the reference value. Although the precision of GPS baseline estimations is smallest using the stochastic model of satellite elevation angle-based secant function (Method E), the GPS height component severely deviates from the reference value, indicating a poorer accuracy. Using the satellite elevation angle-based cosine function, not only the GPS baseline component, especially GPS height, is closest to the reference values, but the precision is also obviously improved.

And therefore, the stochastic model of satellite elevation angle-based cosine function has the best performance, and is proposed to be used in the GAMIT.

Acknowledgments. The authors would like to thank Dr. Bob King and Dr. Peng Fang for their valuable discussions and help in this study. We also thank Dr. Kosuke Heki, Dr. S. Miyazaki and an anonymous reviewer for useful comments and improvements on the manuscript.

\section{References}

Barnes, J. B., N. Ackroyd, and P. Cross, Stochastic modeling for very high precision real-time kinematic GPS in an engineering environment. XXI International Congress of FIG, Brighton, UK, 19-25 July, 1998.

Blewitt, G., GPS Data Processing Methodology: From Theory to Applications, in GPS for Geodesy, edited by P. J. G. Teunissen and A. Kleusberg, p. 231-270, Springer-Verlag, Berlin, 1998.

Bona, P., Precision, cross correlation, and time correlation of GPS phase and code observations, GPS Solutions, 4(2), 3-13, 2000.

Brunner, F. K., H. Hartinger, and L. Troyer, GPS Signal Diffraction Modelling: The Stochastic SIGMA- $\Delta$ Model, Journal of Geodesy, 73, 259 267, 1999.

El-Rabbany AE-S, The effect of physical correlations on the ambiguity resolution and accuracy estimation in GPS differential positioning. Tech Rep 170, Department of Geodesy and Geomatics Engineering, University of New Brunswick, 1994.

Han, S. and C. Rizos, Standardization of the variance-covariance matrix for GPS rapid static positioning, Geomat. Res. Aust., 62, 37-54, 1995.

Hopfield, H. S., Two-quartic tropospheric refractivity profile for correcting satellite data, J. Geophys. Res., 74(18), 4487-4499, 1969.

Hugentobler, U., S. Schaer, and P. Fridez, BERNESE GPS Software Version 4.2, Astronomical Institute, University of Bern, 2001.

Jin, S. and J. Wang, Impacts of stochastic models on GPS-derived ZTD estimations. 17th Int. Tech. Meeting of the Satellite Division of the U.S. Institute of Navigation, Long Beach, California, 21-24 September, 2004. 
Jin, X. and C. D. de Jong, Relationship between satellite elevation and precision of GPS code observations, The Journal of Navigation, 49, 253-265, 1996.

King, R. W. and Y. Bock, Documentation for the GAMIT GPS Analysis Software, Mass. Inst. of Technol., Cambridge Mass, 1999.

Rizos, C., Principles and Practice of GPS Surveying Monograph 17, School of Geomatic Engineering, the University of New South Wales, 555 pp., 1997.

Saastamoinent, J., Contribution to the theory of atmospheric refraction, Bulletin Geodesique, 107, 13-34, 1973.

Satirapod, C., J. Wang, and C. Rizos, Modelling Residual Systematic Errors in GPS Positioning: Methodologies and Comparative Studies, In Vistas for Geodesy in the New Millennium, edited by J. Adams and K. P. Schwarz, IAG Symp. Vol. 125, pp. 410-414, Springer-Verlag, ISBN 3-540-43454-2, 2002.

Satirapod, C., J. Wang, and C. Rizos, Comparing different GPS data pro- cessing techniques for modelling residual systematic errors, J. Surv. Eng., 129(4), 129-135, 2003.

Tiberius, C. and F. Kenselaar, Variance component estimation and precise GPS positioning: case study, J. Surv. Eng., 129(1), 11-18, 2003.

Wang, J., Stochastic Assessment of GPS Measurements for Precise Positioning, 11th Int. Tech. Meeting of the Satellite Division of the U.S. Inst. of Navigation, Nashville, Tennessee, 15-18 September, 81-98, 1998a.

Wang, J., M. Stewart, and M. Tsakiri, Stochastic modeling for static GPS baseline data processing, J. Surv. Eng., 124(4), 171-181, 1998b.

Wang, J., C. Satirapod, and C. Rizos, Stochastic assessment of GPS carrier phase measurements for precise static relative positioning, Journal of Geodesy, 76(2), 95-104, 2002.

S. Jin (e-mail: sgjin@kasi.re.kr), J. Wang, and P.-H. Park 